PALEO

Revue d'archéologie préhistorique

$13 \mid 2001$

Varia

\title{
« Le Sansonnet » et « Les Agnels » (Vaucluse), un exemple de fragmentation thermique intentionnelle du silex au Sauveterrien
}

"Le Sansonnet", and "Les Agnels" sites (Vaucluse) : heat treatment for fracturing of flint blocks

\section{Raphaële Guilbert}

\section{OpenEdition \\ Journals}

Édition électronique

URL : http://journals.openedition.org/paleo/1089

DOI : $10.4000 /$ paleo.1089

ISSN : 2101-0420

Éditeur

SAMRA

Édition imprimée

Date de publication : 14 décembre 2001

Pagination : 245-250

ISSN : $1145-3370$

Référence électronique

Raphaële Guilbert, « «Le Sansonnet » et « Les Agnels » (Vaucluse), un exemple de fragmentation thermique intentionnelle du silex au Sauveterrien », PALEO [En ligne], 13 | 2001, mis en ligne le 26 mai 2010, consulté le 07 juillet 2020. URL : http://journals.openedition.org/paleo/1089 ; DOI : https:// doi.org/10.4000/paleo.1089

Ce document a été généré automatiquement le 7 juillet 2020

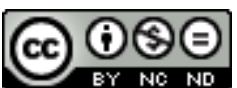

PALEO est mis à disposition selon les termes de la licence Creative Commons Attribution - Pas d'Utilisation Commerciale - Pas de Modification 4.0 International. 


\section{« Le Sansonnet » et « Les Agnels » (Vaucluse), un exemple de fragmentation thermique intentionnelle du silex au Sauveterrien}

"Le Sansonnet", and "Les Agnels" sites (Vaucluse) : heat treatment for fracturing of flint blocks

Raphaële Guilbert

\section{Introduction}

1 Le traitement thermique des roches, et en particulier des roches siliceuses, est un domaine qui intéresse tout particulièrement les préhistoriens. De nombreuses expérimentations ont déjà été tentées pour reproduire et expliquer les changements structuraux du silex. Ainsi la reconnaissance physique du traitement thermique intentionnel, liée à la taille du silex, est établie depuis maintenant plus de trente ans (Crabtree et Butler, 1964, Bordes 1969, Inizan, Roche, Tixier, 1976-1977). Le diagnostic est principalement fondé sur la présence de plages restées mates en contact avec l'air chaud et de plages luisantes sur les produits débités ou façonnés après chauffe. En revanche, l'étonnement des blocs qui consiste à chauffer puis refroidir rapidement une roche pour développer des fissures existantes ou en créer de nouvelles et en faciliter l'éclatement, n'a fait l'objet d'aucun protocole expérimental et reste encore aujourd'hui uniquement documenté par les témoignages ethnographiques (Mandeville 1973). 


\section{Un référentiel ethnographique important}

2 L'éclatement par choc thermique ou le traitement thermique progressif des roches sont assez souvent mentionnés à des degrés de précisions différents dans la littérature ethnographique. M. D. Mandeville a notamment élaboré un travail de recherche sur les témoignages des différents usages de l'action du feu sur les matières minérales au sein des populations de tradition "primitive" (Mandeville 1973). Nous avons sélectionné certains d'entre eux concernant tout particulièrement l'étonnement. C'est ainsi que $\mathrm{H}$. Lehmann capturé en1870 par les Apaches, décrit la pratique de l'étonnement d'énormes blocs de silex sélectionnés ensuite pour le minimum de mise en forme qu'ils requièrent (Lehmann 1927). On apprend avec E. H.Man (Man 1883, p. 380) que les aborigènes de l'île d'Andama (Australie) procèdent de manière identique mais cette fois pour le grès, et que les Viards du nord de la Californie utilisaient également l'étonnement pour obtenir une série d' " éclats" (Power 1877). Les Yuroks, dans la même région des EtatsUnis, éclateraient des blocs diaclasés que faciliterait l'étonnement après refroidissement (Schumacher 1874, p. 356). Enfin, A. P. Elkin relate l'utilisation du choc thermique pour la fracture de petits galets de silex dans le Northern Kimberley, en Australie (Elkin, 1948).

3 En fait, si la pratique de l'étonnement semble assez couramment utilisée et cela pour différentes roches, l'ensemble de ces récits ne donne que peu ou pas d'indications sur les différentes chaînes opératoires. Le type de roche n'est pas toujours mentionné, ni même leurs dimensions. Plus récemment $\mathrm{P}$. Pétrequin, à propos de l'étude des haches de pierre en Irian Jaya (Indonésie), décrit l'éclatement des gros blocs de basalte par choc thermique avant sélection des éclats à arêtes vives et dégrossissage au percuteur de quartzite par percussion directe (Pétrequin 1993).

4 Si le référentiel ethnographique est étoffé, il faut bien admettre en revanche que l'étonnement reste encore mal identifié dans le matériel archéologique. Mais rappelons par comparaison qu'au début des années 60 , la présence du traitement thermique concernant cette fois-ci la chauffe progressive du silex, ne s'est pas d'emblée imposée aux préhistoriens malgré des stigmates plus lisibles que ceux induits par l'étonnement des blocs.

\section{Les données historiques}

Crabtree puis J. Tixier et $\mathrm{F}$. Bordes ont été, dans le milieu des années 60 parmi les premiers à observer des traces de traitement thermique sur des outils préhistoriques (Crabtree cité dans A. Jelinek 1965), sur des fragments de feuille de laurier provenant de Laugerie-Haute en silex Bergeracois (Inizan et al. 1976, Bordes 1969), ou encore sur plusieurs pointes de flèches provenant d'Algérie (mentionné par J. Tixier dans Inizan et al. 1976-1977 et Dedieu 1965). Dix ans plus tard, François Bordes (Bordes 1976) évoque le scepticisme de ses collègues préhistoriens concernant le traitement à la chaleur de certaines pointes de Kimberley provenant de collections australiennes. En fait, si la caractérisation des stigmates de chauffe n'est pas fondamentalement remise en question par les préhistoriens, en revanche, le caractère intentionnel de la chauffe en contexte préhistorique semble poser un problème. 
6 Il faut donc attendre la fin des années 1970 avec la parution du premier article de synthèse (Inizan, Roche, Tixier 1976-1977) qui intègre les principales analyses expérimentales liées au traitement thermique mais aussi les divers exemples ethnographiques rencontrés notamment en Amérique du Nord et dans le nord de l'Afrique pour que soit admis par les préhistoriens le postulat d'un pré-traitement du silex par la chaleur avant les opérations de façonnage ou de débitage en contexte archéologique. Pourtant, de nombreuses expérimentations ont permis de reproduire le phénomène mais aussi de l'expliquer. Dix ans après l'article de D.E. Crabtree et B.R. Butler en 1964 (Crabtree et Butler 1964), B.A. Purdy (Purdy 1974) entreprend une recherche approfondie sur les phénomènes alors inexpliqués de transformations structurelles provoquées par la chauffe qui donnent au silex une meilleure aptitude à la taille et un aspect mat/luisant caractéristique.

7 Aujourd'hui, le traitement thermique des roches n'est plus à démontrer " cette technique est mise en relation avec le débitage par pression qui doit avoir une origine Sibéro-mongole à la fin du Pléistocène " (Inizan et Tixier 2000, p. 23).

\section{Les stigmates d'identification de la technique}

8 La détermination d'un silex chauffé reste assujettie à une évaluation visuelle qui répond à des critères très variés. La qualité du silex, sa porosité, son taux de silice mais aussi l'environnement dans lequel il est chauffé (sable, cendre, sciure), son taux d'humidité sont autant de paramètres qui modifient les stigmates de chauffe. A ce jour, c'est la présence d'un aspect mat en surface et d'un état de surface " gras " des cassures après chauffe qui reste le meilleur indicateur de l'action thermique. Ce lustré apparaît en tre $200^{\circ} \mathrm{C}$ et $400^{\circ} \mathrm{C}$ selon les types de silex mais $160^{\circ}$ suffisent pour le silex de Bergerac (Inizan, Roche, Tixier 1976-1977). Les expérimentations de S. Binder sur le silex du Vaucluse (Bédoulien) ont permis de constater l'apparition d'un lustré à $200^{\circ}$ (Binder com. personnelle).

9 La rubéfaction est un autre critère de détermination qui varie également et dépend du taux de fer contenu dans le silex. Cette oxydation du fer qui se produit à la chauffe est un phénomène connu et largement décrit dans plusieurs articles (Mandeville 1973, Purdy 1974, Masson 1984, Binder et Pelegrin 1983). "Les changements de coloration sont toujours aisément perceptibles : les silex riches en fer se rubéfient plus ou moins si la chauffe se réalise en atmosphère oxydante (four, air libre). "L'intensité de cette rubéfaction révèle pour différents silex leur richesse respective en fer" (Masson 1984, p.32).

10 En dehors des critères visuels, différentes techniques ont été mises en place pour établir avec certitude le traitement thermique : diffractométrie des rayons $\mathrm{X}$, analyse des traces de fission, mesure de la thermoluminescence, résonance électronique de Spin, analyse thermique différentielle (Olausson et Larsson 1982, Olausson 1983, Robins et al. 1978). Elles restent toutes discutables en particulier quand la chauffe est de faible intensité. 


\section{Les données archéologiques}

11 En Vaucluse, l'étude du matériel provenant de deux sites sauveterriens a permis de mettre en évidence une série d'indices laissant supposer la pratique d'une fragmentation du silex par choc thermique. Cette pratique qui reste à ce jour singulière au moins pour le Sauveterrien, mérite donc d'être décrite. Il conviendra d'en démontrer le caractère intentionnel ou tout au moins d'en donner un début de démonstration en préliminaire à une série d'expérimentations.

Le site du Sansonnet (Crillon le Brave, Vaucluse) (fig nº), fouillé par J. Buisson Catil (Buisson Catil et al.1999) se trouve à proximité d'un affleurement de silex crétacé. Ce dernier est donc abondant et se présente sous forme de gros rognons pluridécimétriques. L'étude de l'industrie lithique a mis en évidence la présence de plusieurs chaînes opératoires orientées vers la production de supports différenciés (1) -d'une chaîne opératoire de petits éclats aux modules assez proches de ceux recherchés pour les microlithes et obtenus à partir de nucléus à plans de frappe multiples, (2) -une chaîne opératoire de petites lamelles débités à partir de nucléus unidirectionnels, et enfin (3) -une production d'éclats plus épais détachés à partir d'un débitage orthogonal (Guilbert 2001). Au sein de cette industrie, de nombreux éclats et lamelles se distinguent par leur brillance. Mais, parallèlement, cet aspect est graduel et les pièces considérées comme douteuses n'ont donc pas été retenues. En revanche, vingt-six nucléus sur trente-sept au total ont incontestablement subi l'action du feu. Une dizaine de blocs non exploités ont été également séparés lors d'un choc thermique (fig ${ }^{\circ} 2$ ). L'un de ces blocs présente d'une part une rubéfaction intense qui homogénéise en rouge orangé la couleur de ses deux parties, d'autre part une série de négatifs d'éclats parasites luisants qui viennent contraster avec l'aspect globalement mat de la surface (fig. $n^{\circ} 2$ ). De plus, la partie sous corticale présente une zonation intense. Ici les oxydes ont migré en périphérie, formant un liseré très rouge sous cortical. La montée en température ou le refroidissement trop rapide a déstructuré la matrice de deux autres blocs. Leur surface non débitée est lisse. Une fois testés, les négatifs d'éclats laissent apparaître une surface accidentée, liée vraisemblablement au mauvais contrôle de la chauffe (fig. $\mathrm{n}^{\circ} 2$ ). Ces derniers exemples sont précieux car ils attestent clairement l'antériorité de la chauffe par rapport au débitage. Ils viennent s'ajouter à l'imposante majorité des nucléus présentant des plages mates et des négatifs luisants, et parfois de grandes zones sur lesquelles on peut observer des séries d'ondes concentriques autour d'un noyau plus mat. 
Figure 1 : Localisation des sites du Sansonnet, et des Agnels. Figure 1 : Situation of Sansonnet and Agnels sites.

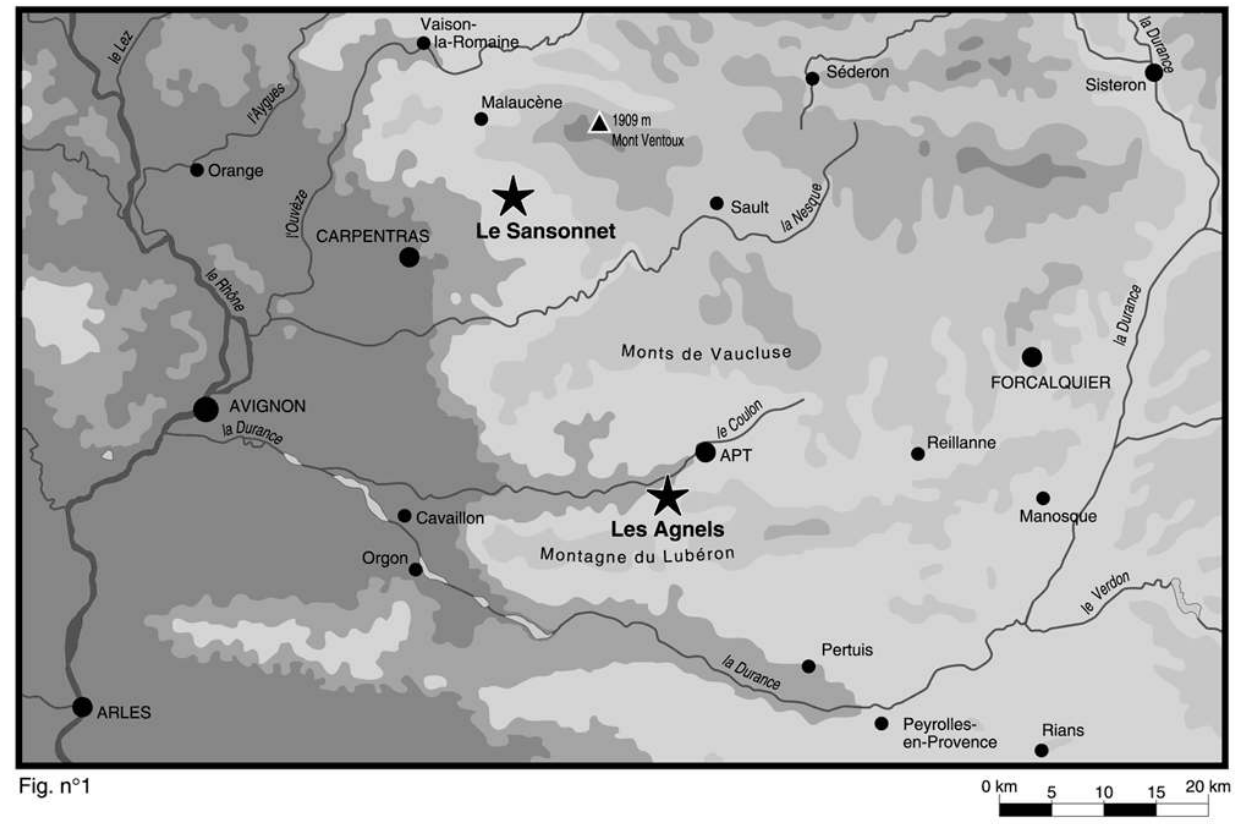


Figure 2 : Pièces chauffées du site Le Sansonnet (Crillon, Vaucluse). Figure 2 : Fire treated flints of Sansonnet site (Crillon, Vaucluse).
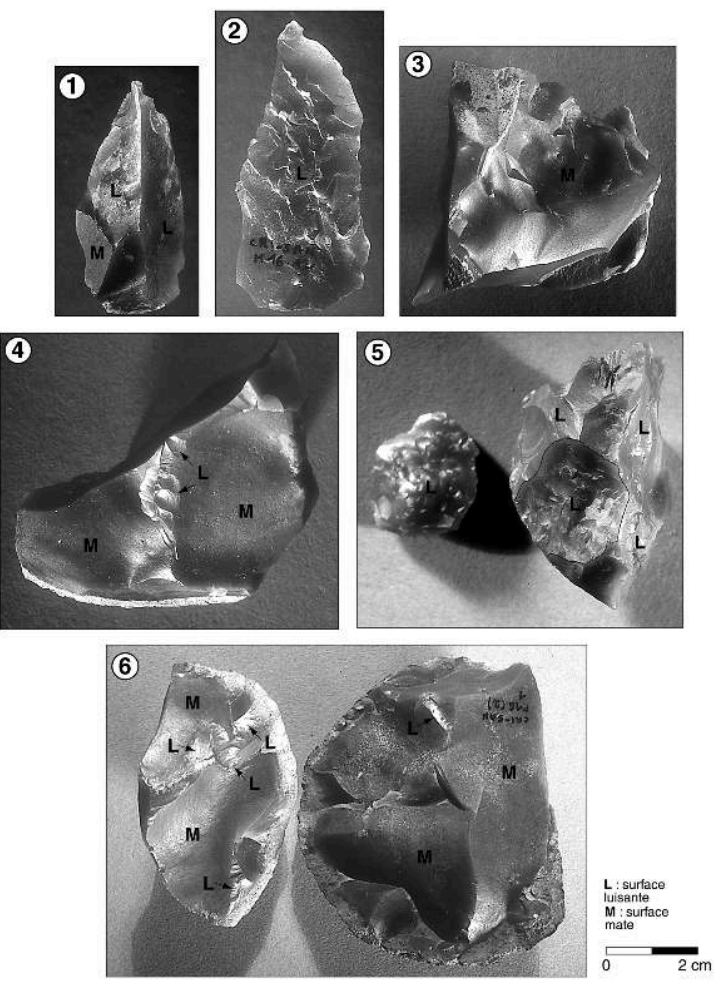

1- éclat lamellaire avec plage mate. 2- face inférieure d'éclat lamellaire débité à partir d'un bloc trop chauffé sur lequel on observe des ondulations irrégulières. 3 et 4- éclats étonnés sur lesquels on distingue des zones mates et des négatifs luisants d'éclats parasites. 5- remontage d'un nucléus bifacial avec un éclat dont la face inférieure révèle une chauffe mal maîtrisée. 6- remontage de deux blocs étonnés par le feu.

1 - Flake with dull zone. 2- Irregular ripples on the ventral face of a flake knapped from too heated core. 3 et 4 -Flakes removed by fire with dull zones and bright surfaces of secondary micro removals (L). 5- Refitting of flakes and a bifacial core too much heated. 6- Rifitting of two blocks removed by fire.

En revanche, l'effectif des éclats pour lesquels l'action du feu ne fait aucun doute, reste faible (une dizaine de pièces au total). Pourtant les trois-quarts des nucléus attestent de son utilisation fréquente. Il s'agit d'éclats extrêmement luisants ou présentant une face inférieure irrégulière caractérisée par de multiples décrochements, qui attestent un mauvais contrôle de la chauffe.

Le deuxième site sauveterrien étudié : les Agnels (Bonnieux, Vaucluse) (fig $n^{\circ} 1$ ), fouillé par M. Livache (Livache 1984), témoigne également de cette pratique sur les blocs de silex. Ce gisement compte plusieurs types de matières premières, mais l'action du feu n'est clairement repérée que sur l'une d'entre elles. Il s'agit d'un silex éocène bréchique à texture très hétérogène. Une partie de ce silex est de mauvaise qualité l'autre est au contraire très siliceux et présente un grain très fin. Notons au passage qu'un nucléus atteste de la concomitance de ces deux aspects. Encore une fois, la détermination des stigmates s'effectue plus aisément sur les pièces de plus gros module qui gardent des plages mates résiduelles.

Ainsi, de toute évidence la difficulté du diagnostic réside dans l'intensité des stigmates. Or en ce qui concerne les blocs du Sansonnet, les contrastes entre plages mates et surfaces luisantes après débitage, sont peu marqués. Les stigmates sont beaucoup moins développés que ceux liés à une chauffe progressive. Par ailleurs, il se peut que 
l'éclatement au feu soit assez rapide. Le passage bref au feu diminue la netteté des stigmates de chauffe, et en l'occurrence le contraste entre plages mates et plages luisantes.

16 Ajoutons à ce constat qu'un certain nombre de silex ne répond pas de la même manière aux actions du feu. Les quelques expériences personnelles effectuées sur du silex tertiaire permettent de constater un faible développement des plages luisantes (Guilbert en préparation).

\section{Evaluation du caractère intentionnel}

17 Constater la présence de l'étonnement des blocs de silex comme préambule au débitage induit une réflexion sur le niveau de prédétermination de ces populations de chasseurscueilleurs, qu'il reste à démontrer. On peut penser qu'au Sansonnet ces blocs ont été déposés malencontreusement au bord du foyer, et qu'ils ont été ensuite débités de manière complètement opportuniste. Toutefois, si cela était le cas, tout porterait à croire que l'effectif des pièces brûlées serait beaucoup plus important qu'il ne l'est dans le cas présent (une dizaine au total sur plus de deux mille pièces). Par ailleurs, rappelons que le diagnostic est établi à partir de plus des trois-quarts des nucléus, il ne s'agit donc pas de cas isolés. Bien sûr si ces éléments ne prouvent pas l'intentionnalité, ils réduisent la part d'opportunité.

D'un point de vue culturel, on peut concevoir cette pratique de fragmentation et d'obtention de petits blocs comme le prolongement d'un procédé couramment rencontré dans les séries de l'Epigravettien terminal. Cet horizon culturel se caractérise par l'obtention de produits à partir de nucléus sur éclat (Binder 1980).

Dans ce cas précis, l'étonnement du bloc comme celle de l'obtention de l'éclat support permet de réduire, voire d'éviter les premières phases de mise en forme, les séries de dièdres sur les fragments étonnés ou le dièdre de l'éclat détaché, permettant d'emblée l'exploitation du nucléus.

20 Quoi qu'il en soit, si une production de fragments est obtenue par éclatement des blocs, cette technique reste néanmoins relativement aléatoire et impose une importante sélection. C'est probablement la raison pour laquelle de nombreux fragments de silex sont restés bruts de débitage. De surcroît ce tri suppose un dernier facteur qui est celui de l'abondance des disponibilités en matériaux siliceux. Le diagnostic a été possible au Sansonnet car nous possédions à la fois la totalité du matériel, des remontages et des blocs de silex ramassés en prospection. C'est donc l'analyse des chaînes opératoire et des différents aspects de la matière première qui nous a permis de valider l'hypothèse d'une facturation thermique par étonnement de l'industrie au Sansonnet.

21 Si le caractère intentionnel de l'étonnement au cours du Sauveterrien nous semble l'hypothèse la plus probable, il est bien évident que la récurrence de ce phénomène sur plusieurs sites est le moyen le plus sûr pour entériner cette affirmation ${ }^{1}$. Mais parallèlement, il semble nécessaire d'entamer une série d'expérimentations sur l'étonnement. En effet, les diaclases internes du silex du Sansonnet engendrent l'éclatement des blocs assez rapidement. Le silex chauffé des Agnels est, quant à lui, très hétérogène. Ce cas de figure à déjà été rencontré, notamment chez les Yuroks (Schumacher op. cit.). Dans ce cas l'étonnement est facilité par la structure du silex, mais qu'en est-il des autres types de silex plus homogènes?. Actuellement, nous ne 
maîtrisons aucun paramètre concernant les conditions nécessaires et suffisantes à l'éclatement des blocs, que ce soit sur la position des blocs au sein du foyer sur les temps de chauffe ou encore sur les températures de chauffe. Une série d'expérimentations programmées, intégrera l'ensemble de ces paramètres. Elle prendra en considération des silex provenant d'étages géologiques différents, afin de tester non seulement leur aptitude à l'étonnement mais aussi le niveau de lisibilité des stigmates caractéristiques d'une fracturation thermique.

\section{BIBLIOGRAPHIE}

BINDER D. 1980 - L'industrie lithique épipaléolithique de l'Abri Martin. Bulletin du Musée d'Anthropologie Préhistorique de Monaco, t.24, p.333-344.

BORDES F. 1969 -Traitement thermique du silex au solutréen, BSPF, 66/ 7 : 197

BORDES F. 1976 - Coup d'œil sur la Préhistoire australienne, Bull. Soc. Prehist. Fr. 73, n6, p. 12-25 BUISSON CATIL J., BROCHIER J.-E., LIVACHE M., GUILBERT R. 1999 - Le gisement de plein air mésolithique du Sansonnet à Crillon le Brave (Vaucluse). Rapport de fouille du conseil général du Vaucluse.

CRABTREE D.E, BUTLER B.R. 1964 - Notes on experiments in flinknapping : Heat treatment of silica materials. Tebiwa $7 / 1: 1-6$.

DEDIEU B. 1965 - La grotte du Djebel Zabaouine, Libyca, A.P.E., 13, p.99-126.

ELKIN 1948 - Pressure Flaking in Northern Kimberley. Manking, t.148, p.110-113

GUILBERT R. 2001 - Gestion des industries lithiques mésolithiques du sud est de la France. Thèse de l'Université de Paris I Panthéon-Sorbonne. 369, 53pl, 68fig, 63 tab.

INIZAN M.L., ROCHE, TIXIER J. 1976-1977 - Avantage d'un traitement thermique pour la taille des roches siliceuses. Quaternaria, 1976-1977, t. 19, p. 1-17.

INIZAN M.L.,TIXIER J. 2000 - L'émergence des arts du feu : le traitement thermique des roches siliceuses. Paléorient, vol 26/2, p. 23-36.

JELINEK A. J. 1965 - Lithic Technology Conference, les Eyzies, France, American Antiquity, vol. 31, $\mathrm{n}^{\circ} 2, \mathrm{p} .277-279$

LEHMANN H. 1927 - Nine years among the Indians (1870-1879). In Von Boekmann-Jones (Ed.) . Austin, 1927. p.93-94.

LIVACHE M. 1984 - Les Agnels (Vaucluse). Rappport de fouille

MAN E.H. 1883 - On the Aboriginal Inhabitants of the Andaman Islands. The Journal of the Anthropological, Institute of Great Britain and Ireland, 1883, t. 12, p. 379-391.

MANDEVILLE M.D. 1973 - A consideration of the thermal pretreatment of chert. Plains Anthropologist, 1973, t. 1, n.61, p.177-200. 
MASSON A. 1981 - Pétroarchéologie des roches siliceuses. Intérêt en Préhistoire. Université Claude Bernard -Lyon I, 1981. Thèse de 3è cycle.

MASSON A. 1984 - Un point de technologie chasséenne. Nouv.Arch.Mus.Hist. Nat. Lyon, fasc. 22, supp., p.31-42

OLAUSSON D.S, LARSSON L. 1982 - Heat treatment of flint in the Scandinavian stone age?

M.L.U.H.M. New Series $4: 5-25$.

OLAUSSON D.S. 1983 - Experiments to investigate the effects of heat treatment on use-wear on flint tools. Proceeding of the Prehistoric Society 49, p. 1-13.

PETREQUIN P. 1993 - Ecologie d'un outil: la hache de pierre au Irian Jaya (Indonésie) Monographie du CRA 12, CNRS Edition.

POWER S. 1877 -Tribes of California. Contributions to North American Ethnology, t.3, ch.XI, p.104.

PURDY B.A. 1974 - Investigations concerning the therminal alteration of silica minerals : an archaeological approach. Tebina, t.17, p.37-66

PURDY B.A.,BROOKS H.K. 1971 - Thermal alteration of silica minerals, an archaeological approach. Science, 1971, 73, p. 322-325.

SCHUMACHER P. 1874 - Remarks on the kjokken-modding on the Northerwest Coast Smithsonian. Institution Annal Reports, p354-362.

\section{NOTES}

1. J'ai eu depuis, l'occasion de constater sur un site d'Italie du Nord (Colbricon 9) la présence d'un bloc étonné, en partie remonté et sur lequel au moins un des fragments atteste d'un débitage de petits éclats lamellaires. (Guilbert R., Dalméri G. à paraître).

\section{RÉSUMÉS}

L'observation des stigmates de chauffe du silex parallèlement aux remontages partiels de plusieurs blocs, à permis de démontrer la pratique de l'étonnement des blocs (fracturation par choc thermique) pour l'obtention de nucléus sur fragments dans deux ensembles lithiques sauveterriens du Vaucluse. La mise en évidence de cette pratique, marginale, pour cette période, apporte un éclairage nouveau sur les pratiques liées aux modalités de débitage des chasseurs sauveterriens.

Two lithic assemblages, typical of the Sauveterrean period, present characteristic marks of flint blocks fracturing by fire to obtain cores on fragments. The description of this practice, marginal for this period, gives new information on the sauveterrean hunters' methods in flint knapping. 
INDEX

Mots-clés : étonnement par le feu, industrie lithique, Mésolithique

Keywords : fracturing by fire, lithic, Mesolithic

\section{AUTEUR}

\section{RAPHAËLE GUILBERT}

Centre de Recherches Archéologiques, Sophia Antipolis - 06565 Valbonne Cedex. 